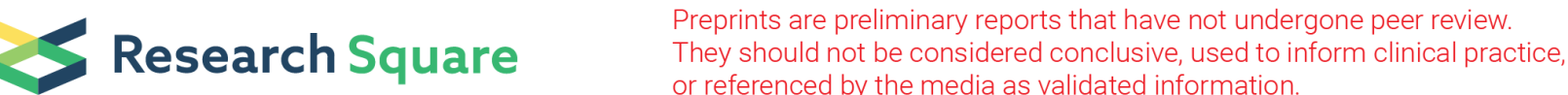 or referenced by the media as validated information.
}

\section{An Iterative Model for Identifying Essential Proteins based on the Whole Process Network of Protein Evolution}

\section{Yaocan Zhu}

Changsha University

Xin Liu

Changsha University

\section{Zhiping Chen}

Changsha University

Zhen Zhang

Changsha University

Lei Wang ( $\nabla$ wanglei@xtu.edu.cn )

Changsha University

\section{Research Article}

Keywords: Essential protein recognition, The whole process network, Protein evolution, Computational methods, Entropy

Posted Date: February 24th, 2022

DOI: https://doi.org/10.21203/rs.3.rs-1362263/v1

License: (c) (i) This work is licensed under a Creative Commons Attribution 4.0 International License. Read Full License 
$4{ }^{1}$ Institute of Bioinformatics Complex Network Big Data, Changsha University, Changsha 410022,

5 China

$6 \quad{ }^{2}$ Big Data Innovation and Entrepreneurship Education Center of Hunan Province, Changsha

7 University, Changsha 410022, China

8 *Correspondence: wanglei@xtu.edu.cn

\section{Abstract}

11 Background: Essential proteins play important roles in cell growth and regulation. However, due to

12 high cost and low efficiency of traditional biological experiments to identify essential proteins, in

13 recent years, with the development of high-throughput technologies and bioinformatics, more and

14 more computational models have been proposed to infer key proteins based on Protein-Protein

15 Interaction (PPI) networks.

16 Result: In order to improve the predictive accuracy of computational methods, a novel prediction

17 model named MWPNPE (Model based on the Whole Process Network of Protein Evolution) was

18 proposed to infer potential basic proteins in this manuscript. In MWPNPE, known PPI data and gene

19 expression data were first downloaded from benchmark databases and adopted to construct the whole

20 process network of protein evolution. And then, considering that the interaction between proteins is a

21 kind of dynamic process, a new measure was designed to estimate the relationships between proteins,

22 based on which, an improved iterative algorithm was put forward to evaluate the importance of

23 proteins. Finally, in order to verify the predictive performance of MWPNPE, we compared it with 
24 state-of-the-art representative computational methods, and experimental results demonstrated that the

25 recognition accuracy of MWPNPE in the top 100, 200 and 300 candidate key proteins can reach 89,

26166 and 233 respectively, which is significantly better than the predictive accuracies achieved by

27 these competitive methods.

28 Conclusions: Through combining a variety of data sources of proteins, a whole process network of

29 protein evolution was constructed first in this manuscript, which greatly reduced the noises and errors

30 existing in original PPI networks. Moreover, through considering the dynamic process of interactions

31 between proteins in the network, a new measure was designed to estimate the relationship between

32 proteins, which improved the predictive accuracy of the newly proposed model effectively. Hence, it

33 can be seen that MWPNPE may be a useful tool for the development of key protein recognition in the 34 future.

35 Keywords: Essential protein recognition, The whole process network, Protein evolution, 36 Computational methods, Entropy

\section{$37 \quad$ Background}

38 Essential proteins are the basis of cell and tissue structure [1]. However, it is time-consuming

39 and high-costly to infer essential proteins by traditional ways such as gene knockout [2] and RNA

40 interference (RNAi) [3-4], etc. In recent years, with the rapid accumulation of PPI data and other

41 biological data of proteins [5-6], a lot of computational models have been proposed to infer potential

42 key proteins and achieved great advances in the improvement of predictive performance. For 43 example, based on the concept of centrality, a series of different methods, including SC [7], EC [8],

44 DC [9] (degree centrality), BC [10] (better centrality), CC [11] (closeness centrality), NC [12]

45 (neighbor centrality) and IC [13] (information centrality), were proposed successively by adopting 46 different topological characteristics of the PPI networks. In addition, based on the local features of 
PPI networks, Qi et al and Li et al proposed a calculative model named LID [14] (Local Interaction

Density) and LAC [15] (Local Average Connection) respectively. Lin et al designed two kinds of prediction model named MNC (Maximum Neighborhood Connectivity) and DMNC (Maximum Neighborhood Connectivity of Density) separately [16]. Jeong et al introduced a central lethal rule to estimate the relationships between proteins as well [17]. However, due to a variety of noise data such as false positive and false negative existing in original PPI networks [18-19], satisfactory accuracy cannot be achieved by these methods based solely on PPI networks in the identification of essential proteins. Thus, in order to further improve the predictive accuracy of computational models, some new methods have been proposed by integrating PPI networks with biological information of proteins. For instance, Zhang et al put forward a calculation method by fusing dynamic PPI networks [20]. Lei et al designed a prediction model based on the artificial fish swarm optimization [21]. Zhao et al designed a model by combining the gene expression profiles of proteins and PPI networks [22]. Zhang et al proposed a detection model called CoEWC by integrating topological properties of PPI networks and the gene co-expression characteristics of proteins [23]. Ren et al introduced a method called ECC by integration of PPI network topology and protein complexes [24]. Li et al established a model called PEC based on the combination of protein-protein interaction and gene expression data [25]. Sun et al proposed an iterative method called IoMCD based on the cross entropy to fuse biological information [26]. Li et al presented an iterative method named CVIM based on the fusion of protein topology and functional features [27]. Peng et al designed an iterative method called ION by integrating biological information and PPI network characteristics [28]. Zhang et al defined a

67 predictive method called TEGS based on the combination of subcellular location information and PPI network structure [29-30]. Lei et al designed a model named RSG by combining RNA SEQ data, go annotation and subcellular localization [31]. Zhao et al proposed an iterative calculation method called RWHN [32] through integrating protein domain information, subcellular localization information and protein homology information with topology features of PPI networks. Experimental 
results show that these methods based on the combination of biological information of proteins and

73 topological characteristics of PPI networks can obtain much better prediction accuracy.

74 Inspired by above models, in this manuscript, a novel iterative method called MWPNPE was

75 designed based on a newly constructed whole process network of protein evolution to infer potential

76 essential proteins. In MWPNPE, through combining the original PPI network with the conservative

77 degrees and gene expression profiles of proteins, the whole process network of protein evolution was

78 first constructed. And then, through considering the dynamic process of interactions between

79 proteins, a new measure was designed to estimate the relationships between proteins, based on

80 which, an iterative algorithm was proposed to evaluate the importance of proteins. Finally, in order to

81 verify the predictive effects of MWPNPE, we compared it with state-of-the-art prediction models

82 such as SC [7], EC [8], DC [9], BC [10], CC [11], NC [12], IC [13], PEC [25], LAC [15], CoEWC

83 [23], POEM [22], ION [28], TEGS [30] and RWHN [32] simultaneously, and experimental results

84 demonstrated that MWPNPE outperformed all these competitive methods in terms of predictive 85 accuracy of essential proteins.

86 Methods

As shown in Figure 1, the flowchart of MWPNPE consists of the following four major steps:

Step 1: Based on the known PPI data and gene expression data of proteins downloaded from benchmark databases, the whole process network of protein evolution will be constructed first.

90 Step 2: Through considering the dynamic process of interactions between proteins, a new

91 measure will be designed to estimate the relationships between proteins, based on which, topological

92 characteristics including the degrees of compactness, relevance and aggregation will be extracted for

93 proteins from the whole process network of protein evolution in succession. 


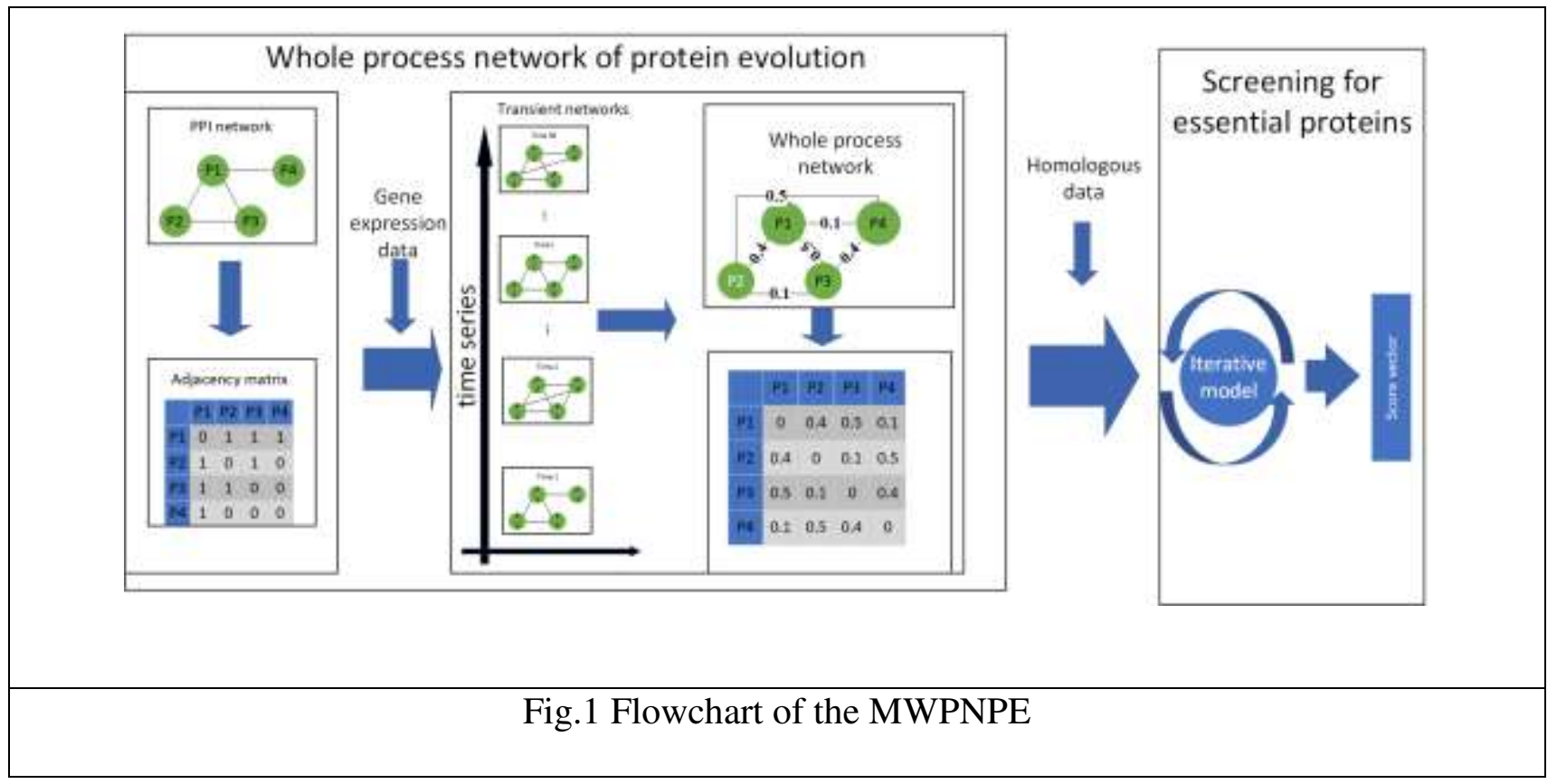

Step 3: Based on the gene expression data and homologous information of proteins, biological

characteristics including the homology fraction and the co-expression intensity will be extracted for 96 proteins as well.

Step 4: Finally, based on the topological and biological characteristics extracted for proteins, an improved iterative method will be designed to estimate the importance of proteins.

99 Construction of the whole process network of protein evolution

100 Construction of original PPI networks and transient networks of proteins

101 Firstly, we will download three datasets of known PPIs from different databases such as Gavin

102 [34], Krogan [35] and DIP [36] respectively. Based on each dataset $\Omega$, let $N_{p}=\left\{p_{1}, p_{2}, \ldots, p_{N}\right\}$

103 denote the set of proteins in $\Omega, E_{p}$ represent the edge set between proteins in $N_{p}$, then we can obtain

104 an original PPI network $O p p i N=\left\{N_{p}, E_{p}\right\}$ as follows: for any two given proteins $p_{i}$ and $p_{j}$ in $N_{p}$,

105 there is $\operatorname{ed}\left(p_{i}, p_{j}\right)=1$ if and only if there is a known interaction between these two proteins in $\Omega$,

106 otherwise, there is $e d\left(p_{i}, p_{j}\right)=0$. 
$108 g_{i 1}, g_{i 2}, \ldots, g_{i T}>$ with $T(=36)$ different time points, where $g_{i t}$ represents the value of the gene

109 expression level of $p_{i}$ at the $t$-th time point, then we can respectively calculate the values of its

110 average gene expression level $\bar{g}_{i}$, its standard deviation $\sigma_{i}$, and the average gene expression level $\bar{g}$

111 of all these $N$ proteins as follows:

$112 \quad \bar{g}_{i}=\frac{1}{T} \sum_{t=1}^{T} g_{i t}$

$113 \quad \sigma_{i}=\sqrt{\frac{1}{T} \sum_{t=1}^{T}\left(g_{i t}-\bar{g}_{i}\right)^{2}}$

$$
\bar{g}=\frac{1}{N} \sum_{i=1}^{N} \bar{g}_{i}
$$

115 For any given protein $p_{i}$ in $N_{p}$, it is obvious that there are fluctuations in concentration of its

116 gene expression level at different time points. For simplicity, supposing that the gene expression

117 value $g_{i t}$ of $p_{i}$ obeys the pseudo normal distribution with a mean (i.e., the mathematical 118 expectation) of $\bar{g}_{i}$ and a variance of ${\sigma_{i}}^{3}$, then we can define the concentration $F_{i}$ of its gene 119 expression value as follow:

$120 \quad F_{i}=\frac{T_{i}}{T}$

121 Where $T_{i}$ represents the number of gene expression value $g_{i t}$ in the horizontal axis interval

$122\left(\bar{g}_{i}-\sigma_{i}{ }^{3}, \bar{g}_{i}+\sigma_{i}{ }^{3}\right)$. Considering that the area in the horizontal axis interval $\left(\bar{g}_{i}-\sigma_{i}, \bar{g}_{i}+\sigma_{i}\right)$ in

123 the standard normal distribution curve is $68.268949 \%$, therefore, in this paper, we will set a threshold

$124 \eta^{\text {Con }}=0.68$, based on which, for any given protein $p_{i}$ at time point $t$, we will divide it into one of

125 these three categories of proteins such as the completely conserved, semi completely conserved or

126 incompletely conserved proteins as follows: 


$$
\left(p_{i}, t\right)=\left\{\begin{array}{cc}
\text { complete } ; \quad \text { if } F_{i} \geq \eta^{\text {Con }} \text { and } \bar{g}_{i} \geq \bar{g} \\
\text { Semicomplete; } & \text { else if } F_{i}<\eta^{\text {Con }} \text { and } g_{i t} \geq \bar{g}_{i} \\
\text { Incomplete } ; & \text { otherwise }
\end{array}\right.
$$

128 According to above formula (5), it is easy to see that the completely conserved proteins refer to

129 proteins with high activity in the whole $T$ time points, the semi completely conserved proteins refer

130 to proteins with high activity at a certain time point or at some time points, while the incompletely

131 conserved proteins refer to proteins with low activity or no gene expression sequence in the whole $T$

132 time points. It is reasonable to believe that proteins are qualified to interact with other proteins only

133 at the time point of high activity. Hence, for any two given proteins $p_{i}$ and $p_{j}$, the interaction

134 between them can be defined as follows:

$$
e_{t}\left(p_{i}, p_{j}\right)=\left\{\begin{array}{c}
0 ; \text { if incomplete }\left(p_{i}\right) \text { or incomplete }\left(p_{j}\right) \\
1 ; \quad \text { otherwise }
\end{array}\right.
$$

137 proteins at the time point $t$, and $E_{t}=\left\{e_{t 1}, e_{t 2}, \cdots, e_{t m}\right\}$ denote the set of protein interactions at the

138 time point $t$, then we can construct 36 different transient networks $\left\{G_{1}, G_{2}, \cdots, G_{t}, \cdots, G_{T}\right\}$ of proteins

139 in final.

\section{Construction of the whole process network $\mathbf{N e t}$}

141 For any given transient network $G_{t}, \forall p_{i} \in N_{p}$, let $N G_{t}\left(p_{i}\right)$ represent the set of adjacent nodes in

$142 N_{p}$ interacting with $p_{i}$ at time point $t$, then there is:

$$
N G_{t}\left(p_{i}\right)=\left\{p_{j} \mid \exists e_{t}\left(p_{i}, p_{j}\right) \in E_{t}, p_{j} \in N_{p}\right\}
$$

Moreover, assuming that $N G_{t}\left(p_{i}\right)=\left\{p_{j}, p_{j+1}, \cdots, p_{j+k}\right\}$, let $T H N G_{t}\left(p_{i}\right)$ represent the set of

nodes in $N_{p}$ interacting with $p_{i}$ or adjacent nodes of $p_{i}$ at the time point $t$, then there is:

$$
T H N G_{t}\left(p_{i}\right)=N G_{t}\left(p_{i}\right) \cup N G_{t}\left(p_{j}\right) \cup N G_{t}\left(p_{j+1}\right) \cup \cdots \cup N G_{t}\left(p_{j+k}\right)
$$


147 In the sequence of transient networks of the whole process of protein evolution, it is reasonable

148 to believe that, with the passage of time, the data of proteins in the past time become less prominent,

149 while the links between recently generated proteins will play a greater role. Therefore, considering

150 that proteins will change with time in the process of evolution, it is obvious that the same edge will

151 appear at different times in these transient networks, and the earlier this edge appears, the more its

152 role in protein evolution will change over time. Hence, we can calculate the weight corresponding to

153 each edge by the following way:

154 For any two given proteins $p_{i}$ and $p_{j}$, assuming that the edge between them at the time point $t$ is

$155 e_{t}\left(p_{i}, p_{j}\right)$, then its weight $D_{t}\left(p_{i}, p_{j}\right)$ at the time point $t$ can be defined as follows:

156

$$
D_{t}\left(p_{i}, p_{j}\right)=\left\{\begin{array}{c}
e d\left(p_{i}, p_{j}\right) ; \quad t=0 \\
D_{t-1}\left(p_{i}, p_{j}\right) * \gamma+e_{t}\left(p_{i}, p_{j}\right) ; \text { otherwise }
\end{array}\right.
$$

Where $\gamma$ is a parameter with value between 0 and 1, and according to the literature [33], in this paper, we will set the value of the parameter $\gamma$ to 0.9 .

Based on above formula (9), for any given protein $p_{i}$, assuming that $p_{j}$ is a neighbor of $p_{i}$, then

160 we can define the weight $w_{t}\left(p_{i}, p_{j}\right)$ of $p_{j}$ to $p_{i}$ at the time point $t$ as follows:

161

$$
w_{t}\left(p_{i}, p_{j}\right)=\frac{D_{t}\left(p_{i}, p_{j}\right)}{\sum_{p_{k \in} \in^{N G}}\left(p_{i}\right) D_{t}\left(p_{i}, p_{k}\right)}
$$

162 According to above formula (10), it is easy to see that there is $\sum_{N G_{t}\left(p_{i}\right)} w_{t}\left(p_{i}, p_{j}\right)=1$, and $163 w_{t}\left(p_{j}, p_{i}\right)$ may not equal to $w_{t}\left(p_{j}, p_{i}\right)$.

164 Obviously, we have reasons to believe that, for any two given proteins $p_{i}$ and $p_{j}$, when the 165 weight $w_{t}\left(p_{i}, p_{j}\right)$ between them reaches the maximum, the role of the link between them can best 
166 reflect the current relationship between them. Hence, let $w_{\max }\left(p_{i}, p_{j}\right)$ represent the maximum weight

167 between $p_{i}$ and $p_{j}$, then we can obtain a weight matrix $W=\left[w_{i j}\right]_{N \times N}$ as follows:

$168 \quad w_{i j}=\frac{w_{\max }\left(p_{i}, p_{j}\right)}{\sum_{j=1}^{N} w_{\max }\left(p_{i}, p_{j}\right)}$

169 Based on above formula (11), it is obvious that we can fuse those 36 transient networks

$170\left\{G_{1}, G_{2}, \cdots, G_{t}, \cdots, G_{T}\right\}$ of proteins into a whole process network $N e t=\left\{N_{p}, W\right\}$ of protein evolution 171 easily.

\section{Construction of the interaction matrix}

\section{Construction of the interaction matrix based on $\mathrm{Net}$}

174 Considering the dynamic process of protein-protein interactions, for any two given proteins $p_{i}$

175 and $p_{j}$, if there are more protein nodes interacting with both of them in $N e t$, then we have reasons to

176 think that the relationship between them will be more reliable. In this section, for any two given

177 proteins $p_{i}$ and $p_{j}$, we will introduce the following three kinds of network topological features such

178 as $D C$ (Degree of Compactness), $D R$ (Degree of Relevance) and $D A$ (Degree of Aggregation) to

179 better reflect the reliability of the relationship between them:

180

$$
D C\left(p_{i}, p_{j}\right)=\left\{\begin{array}{cl}
\frac{\left|N G\left(p_{i}\right) \cap N G\left(p_{j}\right)\right|+1}{\left|N G\left(p_{i}\right)\right|}: & p_{j} \in N G\left(p_{i}\right) \\
\frac{1}{\left|N G\left(p_{i}\right)\right|}: & \text { otherwise }
\end{array}\right.
$$

181

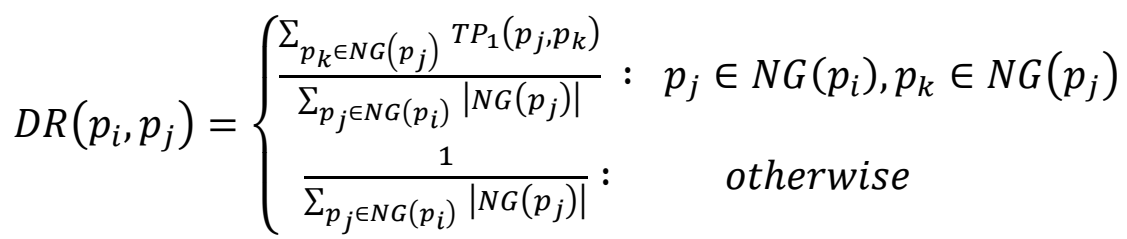

182

$$
D A\left(p_{i}, p_{j}\right)=\left\{\begin{array}{cc}
\frac{\left|T H N G\left(p_{i}\right) \cap N G\left(p_{j}\right)\right|+1}{\left|T H N G\left(p_{i}\right)\right|}: & p_{j} \in N G\left(p_{i}\right) \\
\frac{1}{\left|T H N G\left(p_{i}\right)\right|}: & \text { otherwise }
\end{array}\right.
$$


184 of nodes interacting with $p_{k}$ or adjacent nodes of $p_{k}$ in $O p p i N$, while $|\Lambda|$ is the number of elements

185 in the set of $\Lambda$. As can be seen from the above formulas (12), (13) and (14), for any two proteins $p_{i}$

186 and $p_{j}$ in $N e t$, the more adjacent nodes between them, the greater the value of $\left|N G\left(p_{i}\right) \cap N G\left(p_{j}\right)\right|$

187 will be. Therefore, to a certain extent, $D C\left(p_{i}, p_{j}\right)$ can effectively reflect the tightness between $p_{i}$ and

$188 p_{j}$. Similarly, $\operatorname{DR}\left(p_{i}, p_{j}\right)$ can effectively reflect the degree of correlation between $p_{i}$ and $p_{j}$, and

$189 D A\left(p_{i}, p_{j}\right)$ can effectively reflect the degree of aggregation between $p_{i}$ and $p_{j}$.

190 Hence, for any given protein $p_{i}$, assuming that $p_{j}$ is a neighbor of $p_{i}$, then we can estimate the

191 importance $R\left(p_{i}, p_{j}\right)$ of $p_{j}$ to $p_{i}$ as follows:

192

$$
R\left(p_{i}, p_{j}\right)=w_{i j} *\left(\alpha * D C\left(p_{i}, p_{j}\right)+\beta * D R\left(p_{i}, p_{j}\right)+\varphi * D A\left(p_{i}, p_{j}\right)\right)
$$

Where, $\alpha, \beta$ and $\varphi$ are parameters with values between 0 and 1 , and there is $\alpha+\beta+\varphi=1$.

$$
K\left(p_{i}\right)=\sum_{j=1}^{N} R\left(p_{i}, p_{j}\right)
$$

$$
h_{i j}=\left\{\begin{array}{cl}
\frac{K\left(p_{i}\right)}{\sum_{m=1}^{N} K\left(p_{m}\right)}: & \text { if } i=j \\
\frac{\min \left\{K\left(p_{i}\right), K\left(p_{j}\right)\right\}}{\sum_{m=1}^{N} K\left(p_{m}\right)}: & \text { otherwise }
\end{array}\right.
$$


Based on above formulas (12), (13) and (14), for any given protein node $p_{i}$ in $N e t$, let $T P_{1}, T P_{2}$ and $T P_{3}$ represent the importance of $p_{i}$ based on these three kinds of network topological features such as $D C, D R$ and $D A$ respectively, then we can calculate the values of $T P_{1}, T P_{2}$ and $T P_{3}$ as 206 follows:

$$
T P_{1}\left(p_{i}\right)=\sum_{p_{j} \in N G\left(p_{i}\right)} D C\left(p_{i}, p_{j}\right)
$$

$$
\begin{aligned}
& T P_{2}\left(p_{i}\right)=\sum_{p_{j} \in N G\left(p_{i}\right)} D R\left(p_{i}, p_{j}\right) \\
& T P_{3}\left(p_{i}\right)=\sum_{p_{j} \in N G\left(p_{i}\right)} D A\left(p_{i}, p_{j}\right)
\end{aligned}
$$

210 Based on these $M(=3)$ different biological feature scores obtained above, for all $N$ protein nodes 211 in Net, it is obvious that we can construct a feature matrix $Z$ as follows:

$$
Z=\left[\begin{array}{ll}
T P_{1} \cdots & T P_{M}
\end{array}\right]_{1 \times M}=\left[z_{i j}\right]_{N \times M}
$$

213 According to above formula (21), it is easy to see that, for any given protein node $p_{i}$, there are $M$

214 different features $T P_{1}\left(p_{i}\right), T P_{2}\left(p_{i}\right), \ldots, T P_{M}\left(p_{i}\right)$. Hence, in order to estimate the stability of all 215 these different features, for the $j$-th features $T P_{j}$ of proteins, we will further calculate its weight $\mu_{j}$ 216 based on its entropy $e n_{j}$ as follows:

$$
e n_{j}=-\sum_{i=1}^{N} z_{i j} \ln z_{i j} / \ln N
$$

$$
\mu_{j}=\left(1-e n_{j}\right) / \sum_{i=1}^{M}\left(1-e n_{i}\right)
$$




\section{Calculation of initial scores for proteins}

\section{Calculation of biological feature scores for proteins}

The essentiality of protein has been proved to be closely related to the degree of protein

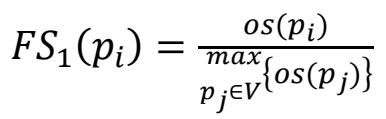

Obviously, the degree of conservatism $F S_{1}\left(p_{i}\right)$ obtained by above formula (24) can be

regarded as one biological feature score of $p_{i}$.

229 Moreover, based on the gene expression sequences of proteins, for any two given proteins $p_{i}$ and $230 p_{j}$, we can further obtain the intensity of co-expression between them by adopting the PCC [45] as 231 follows:

$$
\operatorname{PCC}\left(p_{i}, p_{j}\right)=\frac{1}{T-1} \sum_{t=1}^{T}\left(\frac{\left.g_{i t}-\bar{g}_{i}\right)}{\sigma_{i}^{2}}\right)\left(\frac{\left.g_{j t}-\bar{g}_{j}\right)}{\sigma_{j}^{2}}\right)
$$

$$
F S_{2}\left(p_{i}\right)=\sum_{p_{j} \in N G\left(p_{i}\right)} \operatorname{PCC}\left(p_{i}, p_{j}\right)
$$

\section{Calculation of initial scores based on biological feature scores}

$$
X=\left[F S_{1} \cdots \quad F S_{L}\right]_{1 \times L}=\left[x_{i j}\right]_{N \times L}
$$


240 According to above formula (27), it is easy to see that, for any given protein node $p_{i}$, there are $M$

241 different features $F S_{1}\left(p_{i}\right), F S_{2}\left(p_{i}\right), \ldots, F S_{L}\left(p_{i}\right)$. Hence, in order to estimate the stability of all

242 these different features, for the $j$-th features $F S_{j}$ of proteins, we will further calculate its weight $\theta_{j}$

243 based on its entropy $e n t_{j}$ as follows:

$244 \quad e n t_{j}=-\sum_{i=1}^{N} x_{i j} \ln x_{i j} / \ln N$

$245 \quad \theta_{j}=\left(1-e n t_{j}\right) / \sum_{i=1}^{L}\left(1-e n t_{i}\right)$

246 Thus, based on above formulas, we can calculate initial scores for all these $N$ proteins in Net as 247 follows:

$248 \quad F=\sum_{j=1}^{L} \theta_{j} * F S_{i j}$

249 Algorithm

250 Finally, based on above formulas (17) and (30), let $Y^{0}=F$, then we can calculate the criticality 251 scores for all these $N$ proteins in Net iteratively by the following way:

$252 \quad Y^{t+1}=\tau H Y^{t}+(1-\tau) Y^{0}$

253

Where the parameter $\tau(0 \leq \tau \leq 1)$ is utilized to adjust the proportion of initial score $\left.Y^{0}\right)$ and 254 last iteration score $Y^{t}$.

255 Hence, based on above formula (31), we can present our predictive algorithm MWPNPE as 256 follows:

257

258 Algorithm: MWPNPE

259 
Input: the gene expression data, the original PPI network $O p p i N=\left\{N_{p}, E_{p}\right\}$, the homologous

data, and the parameters $\delta\left(=10^{-5}\right)$ and $K$

262 Output: Top $K$ percent of proteins sorted by the vector $Y$ in descending order

Step1: Constructing 36 different transient networks $\left\{G_{1}, G_{2}, \cdots, G_{t}, \cdots, G_{T}\right\}$ of proteins according to above formula (6).

Step2: Constructing the whole process network $N e t=\left\{N_{p}, W\right\}$ of protein evolution according to above formula (11).

Step3: Constructing the interaction matrix $H$ according to above formula (17).

(30).

Step4: Let $t=0$, calculating initial score vector $Y^{t}$ for all proteins according to above formula proteins.

Step5 : Let $t=t+1$; Calculating $Y^{t+1}$ according to above formula (31).

Step6 : Repeat step 5 until $\left\|Y^{t+1}-Y^{t}\right\|<\delta$.

Step7 : Sorting the values of $Y^{t+1}$ in descending order, and outputting the top $K$ percent of sorted

\section{Results and analysis}

\section{Experimental data}

Considering that Saccharomyces cerevisiae contains rich biological information data and the most complete PPI data, and is widely used in the prediction model for evaluating key proteins, therefore, we will first download known PPIs from the Gavin [34], Krogan [35] and DIP [36] databases respectively, and then, after filtering the repeated interactions and self-interactions from the downloaded datasets, we finally obtain 7669 interactions including 1855 proteins from Gavin, 14317

282 interactions including 3672 proteins from Krogan and 24743 interactions including 5093 proteins 
283 from DIP. Secondly, we will download a benchmark dataset containing 1293 essential proteins in

284 Saccharomyces cerevisiae from the Saccharomyces Genome deletion project database (SGDP) [37],

285 the Saccharomyces Genome Database (SGD) [38], the database of essential genes (DEG) [39] and

286 MIPS [40] to compare predictive accuracy between different recognition models. Moreover, we will

287 download direct homology information data from the Inparanoid database (version 7) [41], and a

288 dataset containing 6776 gene products and 36 samples from the database provided by Tu et al. [42].

289 Finally, based on above newly-downloaded datasets, we will compare MWPNPE with the 14

290 advanced competitive models (as shown in the following Table 1) to estimate its predictive

291 performance based on different measurement and verification methods such as accuracy, folding

292 knife, ROC curve and PR curve, etc. Besides, the influence of parameters $\tau$ on predictive

293 performance of MWPNPE has been evaluated as well.

Table 1. Brief description of 14 advanced competitive prediction models

\begin{tabular}{|l|l|l|}
\hline Algorithm & \multicolumn{1}{|c|}{ Biological information } & \multicolumn{1}{|c|}{ Network topology } \\
\hline SC [7] & NO & Subgraph Centrality \\
\hline EC [8] & NO & Eigenvector Centrality \\
\hline DC [9] & NO & Degree Centrality \\
\hline BC [10] & NO & Betweenness Centrality \\
\hline CC [11] & NO & Closeness Centrality \\
\hline
\end{tabular}




\begin{tabular}{|c|c|c|}
\hline $\mathrm{NC}[12]$ & NO & Neighbor Centrality \\
\hline IC [13] & NO & Information Centrality \\
\hline PEC [25] & Gene expression data & Edge clustering coefficient \\
\hline LAC [15] & NO & Degree Centrality, Common neighbor node \\
\hline $\begin{array}{l}\text { CoEWC } \\
{[23]}\end{array}$ & Gene expression data & Clustering coefficient \\
\hline $\begin{array}{l}\text { POEM } \\
\text { [22] }\end{array}$ & Gene expression data & $\begin{array}{l}\text { Degree Centrality, Subgraph, Edge clustering } \\
\text { coefficient, Closeness Centrality }\end{array}$ \\
\hline ION [28] & Orthologous data & Edge clustering coefficient \\
\hline TEGS [30] & subcellular localization data & Sub-network partition and prioritization \\
\hline $\begin{array}{l}\text { RWHN } \\
{[32]}\end{array}$ & $\begin{array}{l}\text { Orthologous data, subcellular } \\
\text { localization }\end{array}$ & Degree Centrality, protein-domain \\
\hline
\end{tabular}

\section{Comparison between MWPNPE and competitive methods}

295 In this section, we first compared MWPNPE with 13 competitive methods including SC, EC,

296 DC, BC, CC, NC, IC, PEC, LAC, CoEWC, POEM, ION and RWHN based on the Gavin database,

297 and the experimental results were shown in Fig.2. From observing Fig.2, it is easy to see that, in the

298 ranking of true essential proteins inferred by all these competitive prediction methods, MWPNPE can

299 detect more essential proteins in the top 5\%,10\%, 15\%, 20\% and 25\% based on the Gavin database. 
301 are $10.8 \%, 8.6 \%, 12.9 \%, 11.9 \%$ and $11.6 \%$ higher than that of RWHN method respectively.

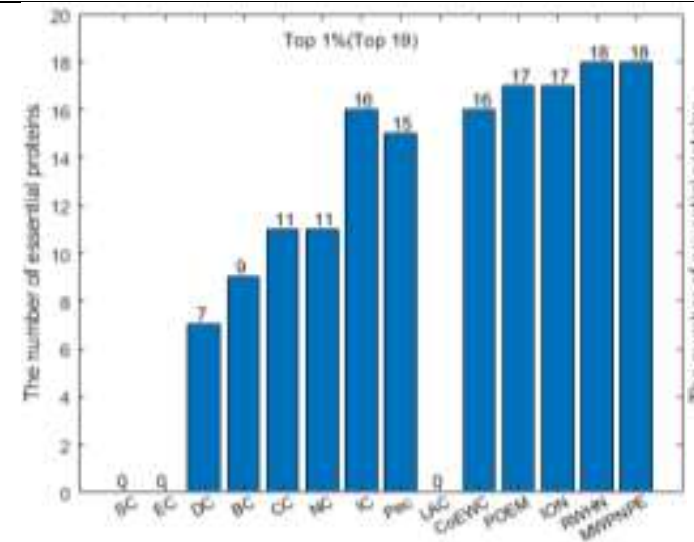

(a)

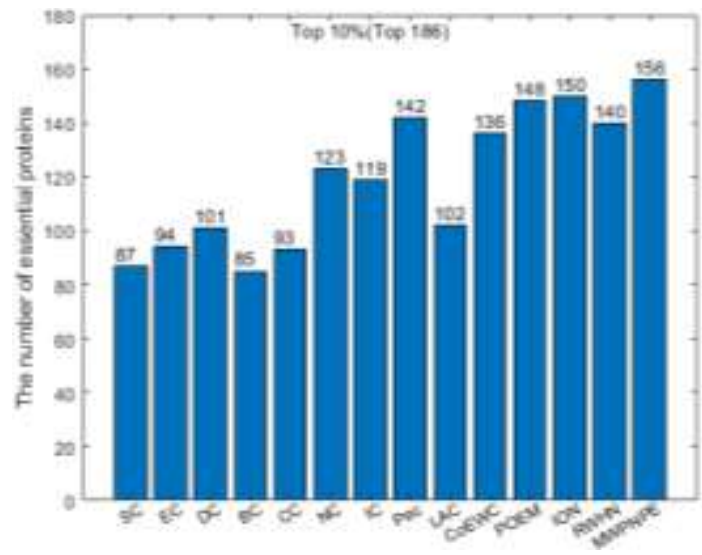

(c)

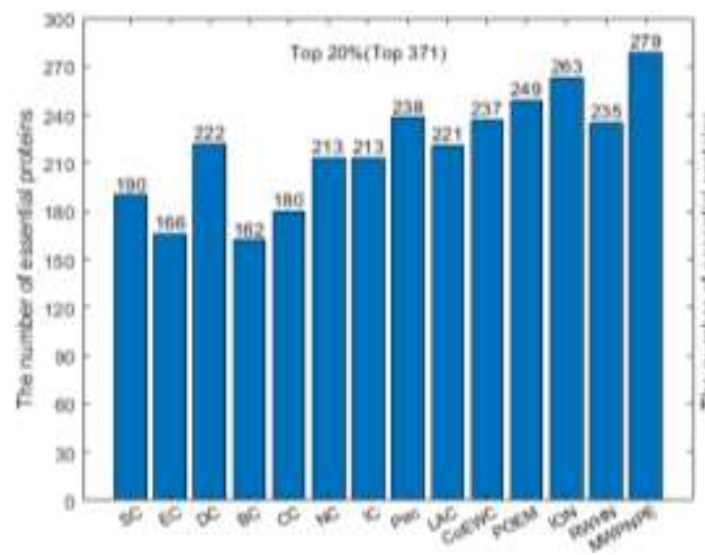

(e)

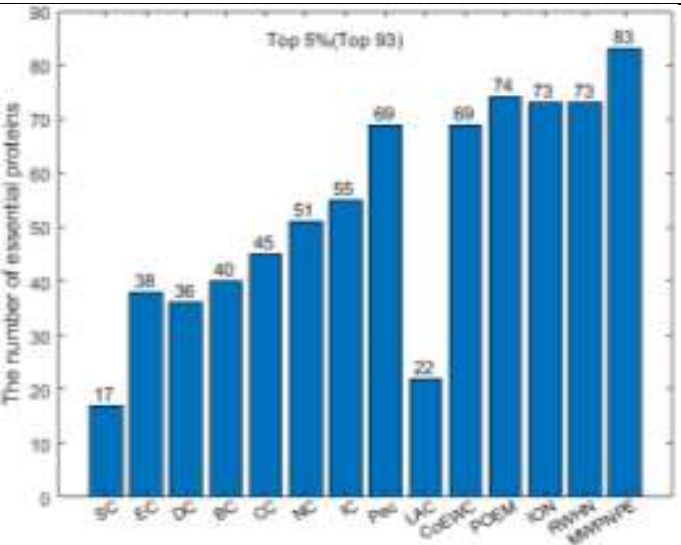

(b)

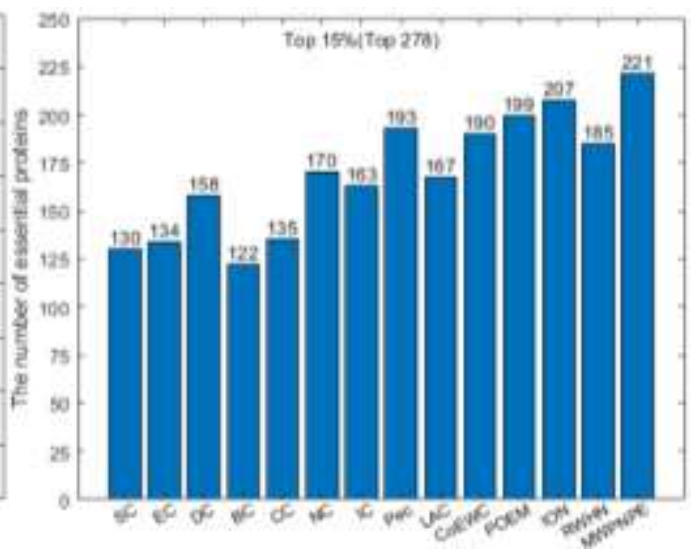

(d)

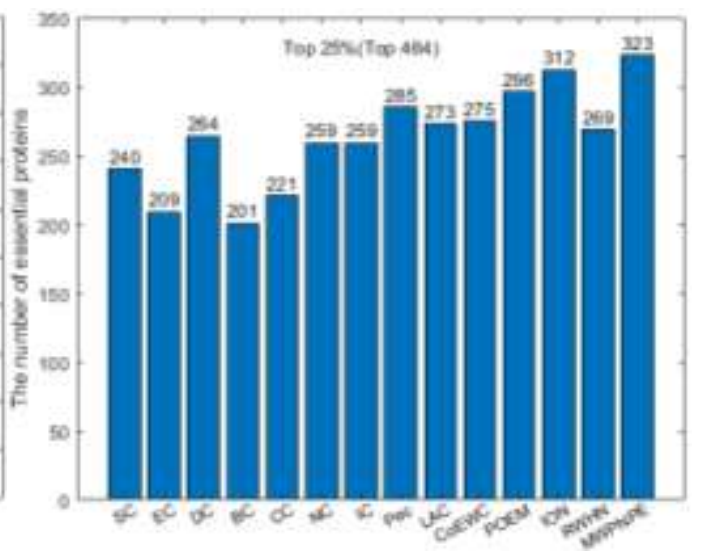

(f)

Fig.2. (a) Top 1\% (19) ranked proteins. (b) Top 5\% (93) ranked proteins. (c) Top 10\% (186) 
ranked proteins. (d) Top 15\% (278) ranked proteins. (e) Top 20\% (371) ranked proteins. (f) Top $25 \%$ (464) ranked proteins. The comparison of numbers of true essential proteins predicted by MWPNPE, SC, EC, DC, BC, CC, NC, IC, PeC, LAC, CoEWC, POEM, ION and RWHN based on the Gavin database.

Next, we compared MWPNPE with 13 competitive methods such as SC, EC, DC, BC, CC, NC,

303 IC, PEC, LAC, CoEWC, POEM, ION and TEGS based on DIP database, and the experimental

304 results were shown in Fig.3. As can be seen from Fig. 3, comparing with these competing methods

305 such as SC, EC, DC, BC, CC, NC, IC, PEC, LAC, CoEWC, POEM, ION and TEGS, the predictive

306 performances of MWPNPE in the top $1 \%$ to $10 \%$ predicted proteins are significantly higher than that

307 of all these competing methods. Especially, the predictive performance of MWPNPE can reach

$30892.2 \%$ in the top $1 \%$ and $79.2 \%$ in the top $5 \%$ predicted proteins.

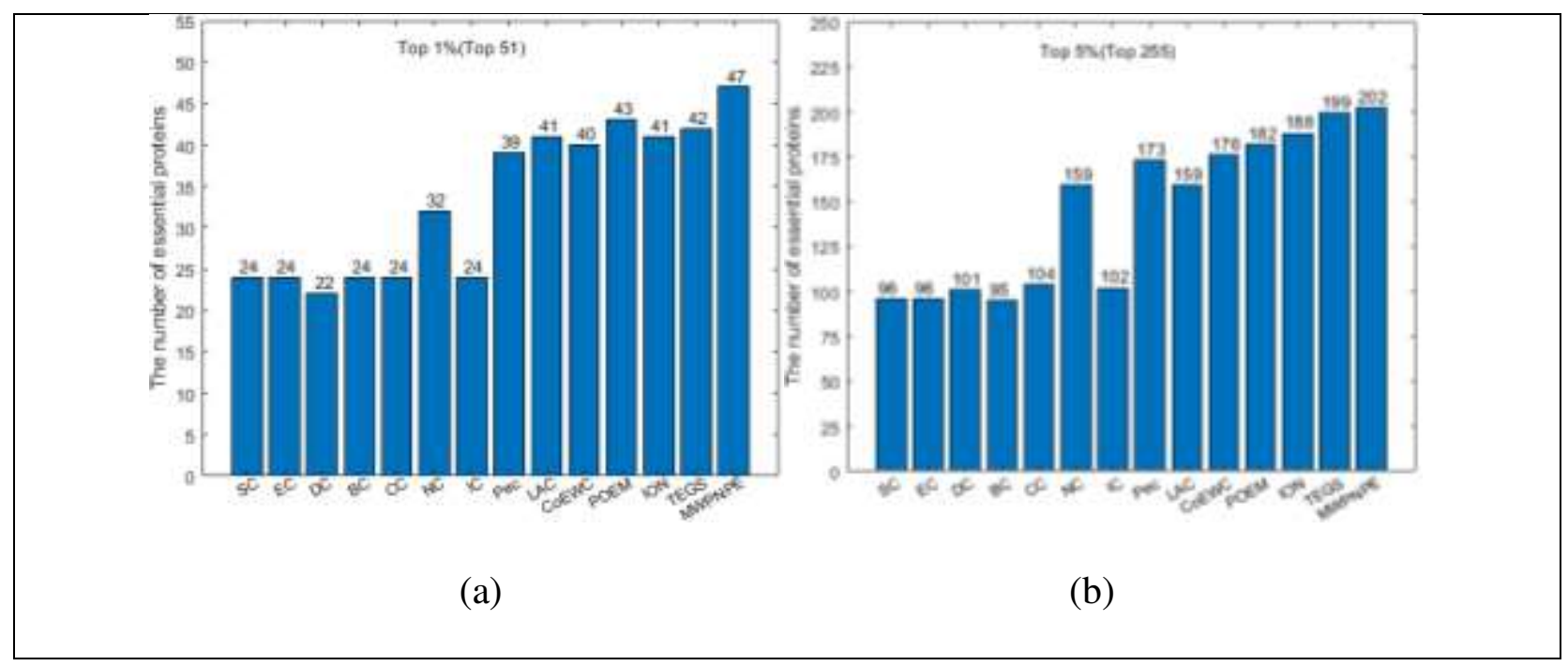




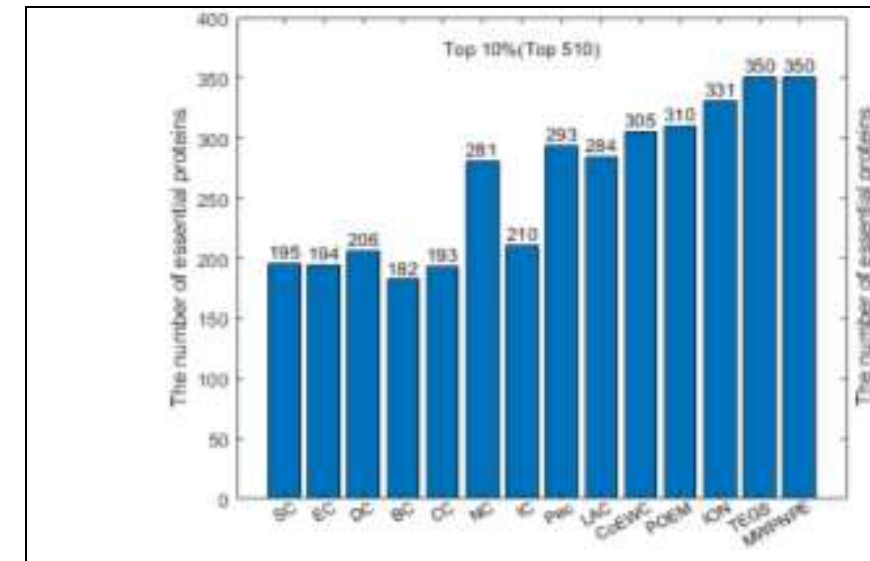

(c)

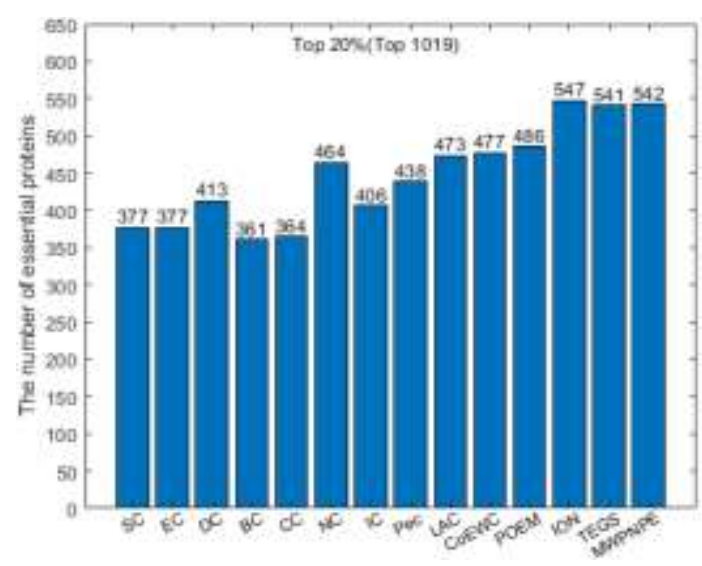

(e)

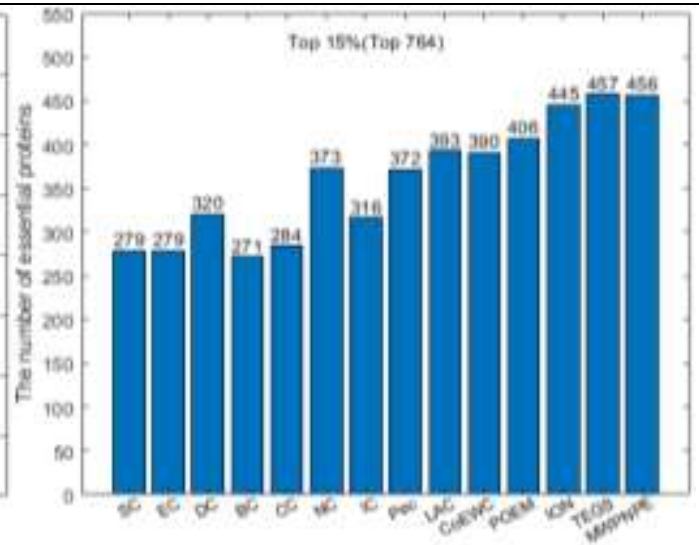

(d)

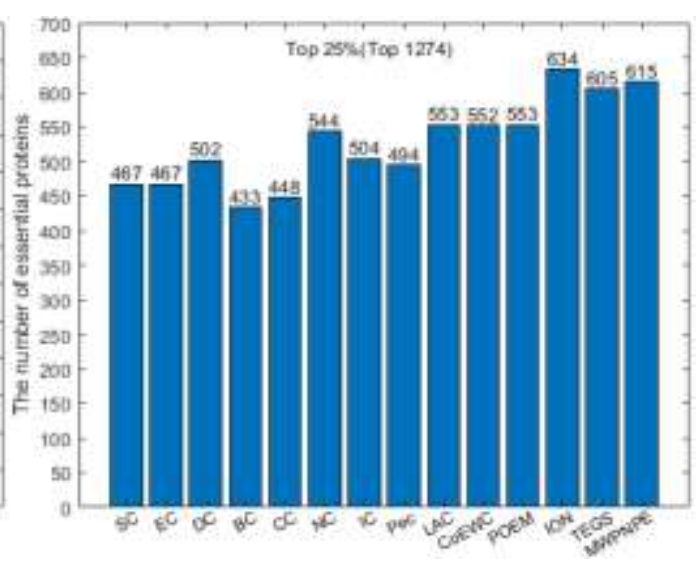

(f)

Fig.3. (a)Top $1 \%$ (51) ranked proteins. (b) Top 1\% (255) ranked proteins. (c) Top 1\% (510) ranked proteins. (d) Top 1\% (764) ranked proteins. (e) Top 1\% (1019) ranked proteins. (f) Top $1 \%$ (1274) ranked proteins. The comparison of numbers of true essential proteins predicted by MWPNPE, SC, EC, DC, BC, CC, NC, IC, PEC, LAC, CoEWC, POEM, ION and TEGS based on the DIP database.

311 results were shown in Table 2. 
312 Table 2 comparison of numbers of true essential proteins predicted by MWPNPE and 10 competing

313 methods based on the Krogan database

\begin{tabular}{|c|c|c|c|c|c|c|}
\hline Methods & Top1\% (37) & Top5\% (184) & Top10\% (367) & $\begin{array}{l}\text { Top } 15 \% \\
(551)\end{array}$ & $\begin{array}{l}\text { Top20\% } \\
(734)\end{array}$ & $\begin{array}{l}\text { Top } 25 \% \\
(918)\end{array}$ \\
\hline $\mathrm{SC}$ & 20 & 78 & 145 & 215 & 273 & 337 \\
\hline $\mathrm{EC}$ & 20 & 78 & 145 & 215 & 273 & 337 \\
\hline $\mathrm{DC}$ & 18 & 98 & 173 & 256 & 321 & 380 \\
\hline $\mathrm{BC}$ & 17 & 83 & 152 & 226 & 286 & 337 \\
\hline $\mathrm{CC}$ & 13 & 68 & 142 & 206 & 262 & 326 \\
\hline IC & 20 & 91 & 173 & 251 & 317 & 378 \\
\hline $\mathrm{Pec}$ & 24 & 123 & 200 & 275 & 333 & 388 \\
\hline LAC & 28 & 119 & 214 & 288 & 344 & 392 \\
\hline CoEWC & 24 & 123 & 215 & 291 & 345 & 401 \\
\hline RWHN & 21 & 97 & 198 & 291 & 382 & 447 \\
\hline MWPNPE & 35 & 140 & 245 & 336 & 410 & 481 \\
\hline
\end{tabular}


315 predictive performance on the basis of Krogan database as well. Specifically, among the top $1 \%$

316 predicted essential proteins, the predictive accuracy of MWPNPE is $42.9 \%, 42.9 \%, 48.6 \%, 51.4 \%$,

$31762.9 \%, 42.9 \%, 31.4 \%, 20.0 \%, 31.4 \%$ and $40.0 \%$ higher than that of SC, EC, DC, BC, CC, IC, PEC,

318 LAC, CoEWC and RWHN separately. And besides, in the top 5\% to $25 \%$ predicted essential

319 proteins, MWPNPE can achieve better predictive performance than all these competitive methods as

320 well.

\section{Validation with jackknife methodology}

322 In this section, we will further adopt validation with jackknife methodology [43] to verify the

323 performance of MWPNPE. Firstly, we extract the top 800 proteins predicted by MWPNPE based on

324 the DIP database to compare MWPNPE with 12 competitive methods including SC, EC, DC, BC,

325 CC, NC, IC, PEC, LAC, CoEWC, POEM and ION, and show the comparison results in Fig.(4). Next,

326 we extract the top 400 proteins predicted by MWPNPE based on the Krogan database to compare

327 MWPNPE with 10 competitive methods including SC, EC, DC, BC, CC, IC, PEC, LAC, CoEWC

328 and RWHN, and show the comparison results in Fig.(5). In both Fig.(4) and Fig.(5), the X-axis

329 represents the number of top ranked proteins, while the Y-axis denotes the number of predicted

330 essential proteins. From observing both Fig.(4) and Fig.(5), it can be clearly seen that the predictive

331 performance of MWPNPE is superior to all these competing models. 


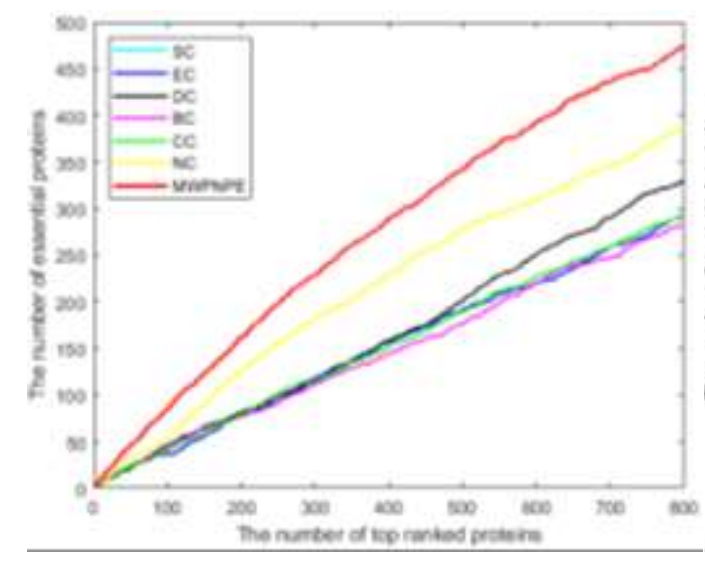

(a)

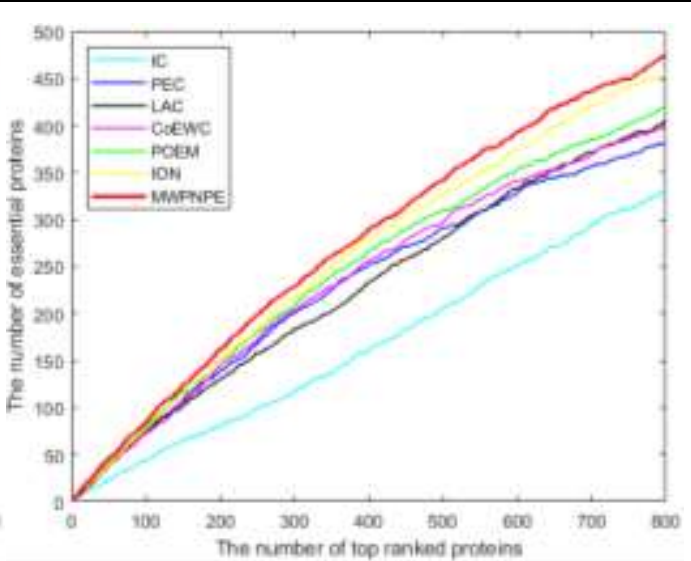

(b)

Fig.4 Comparison of folding knife curve between MWPNPE and 12 competitive methods under the DIP database. (a) Comparison of MWPNPE with SC, EC, DC, BC, CC and NC. (b) Comparison of MWPNPE with IC, PEC, LAC, CoEWC, POEM and ION.

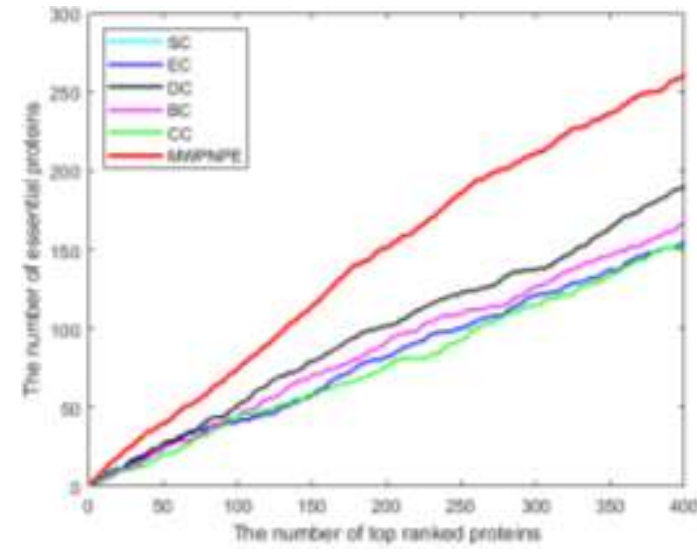

(a)

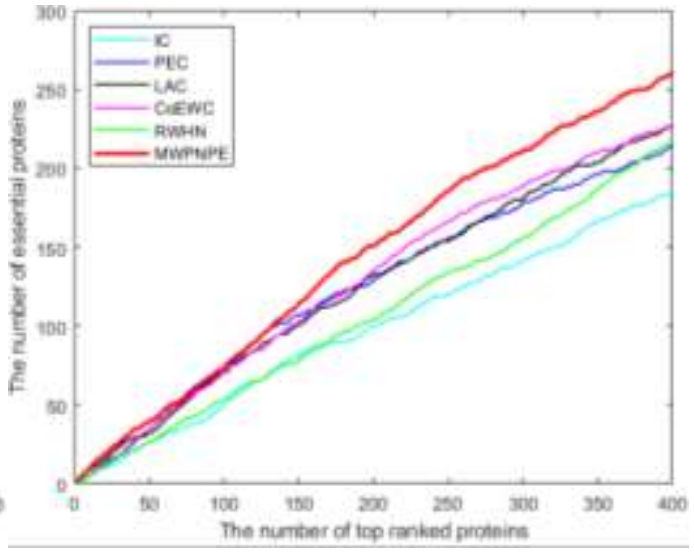

(b)

Fig.5 Comparison of folding knife curve between MWPNPE and 10 competitive methods under the Krogan database. (a) Comparison of MWPNPE with SC, EC, DC, BC and CC. (b) 
Comparison of MWPNPE with IC, PEC, LAC, CoEWC and RWHN.

$333 \quad$ 1.1.1.1 5.4 Validation by precision-recall curves and ROC curves

334 Considering that the proportions of key proteins in databases of Gavin, Krogan and DIP are very

335 small, and researches show that when processing highly sparse datasets, PR curve can provide more

336 information about algorithm performance [44]. Hence, in this section, we will adopt PR (Precision-

337 recall) curve and ROC (receiver operating characteristic) curve to further verify the performance of

338 MWPNPE. Firstly, we will compare MWPNPE with SC, EC, DC, BC, CC, NC, IC, PEC, LAC,

339 CoEWC, POEM and TEGS based on the DIP database. The comparison results are illustrated in Fig.

3406 and table 3. From observing both Fig. 6 and table 3, it is easy to see that the performance of

341 MWPNPE is significantly higher than that of these 12 competitive methods.

342 Next, we will further compare MWPNPE with RTSW, SC, EC, DC, BC, CC, NC, IC, PEC,

343 LAC, CoEWC and RWHN based on the Krogan database. The comparison results are illustrated in

344 Fig. 7 and table 4. From observing both Fig. 7 and table 4, it is easy to see that the performance of

345 MWPNPE is significantly higher than all these 10 competitive methods as well.
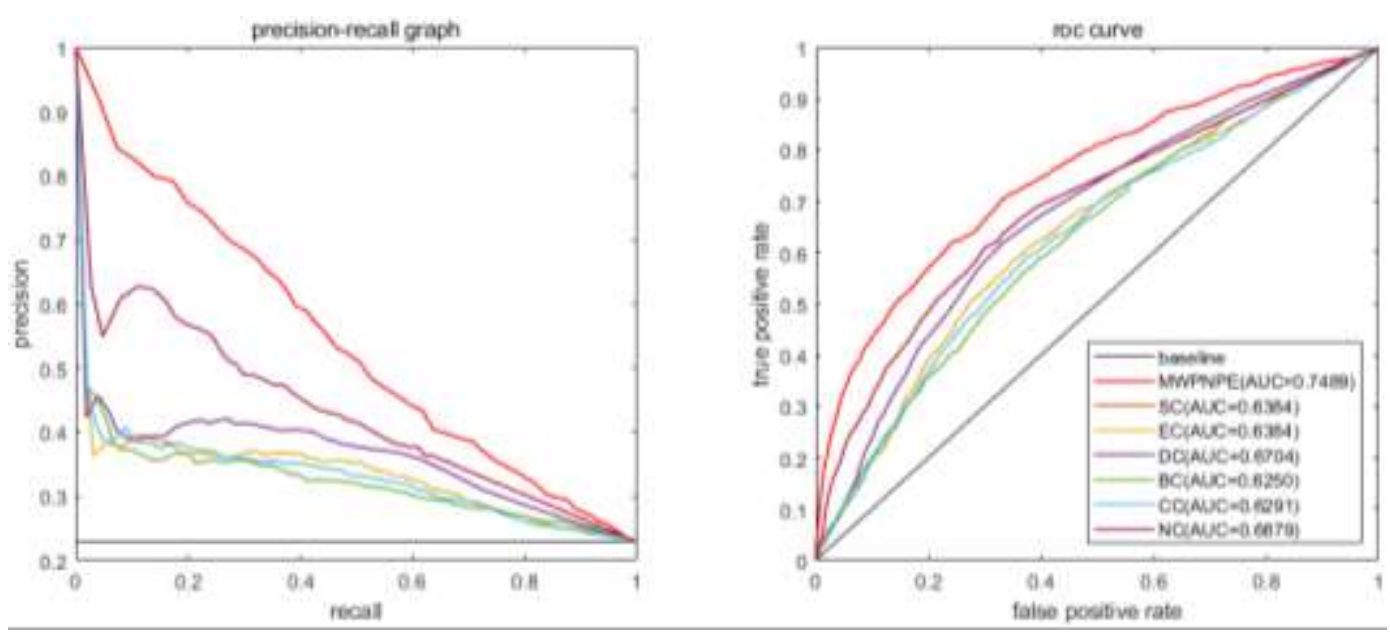

(a) 

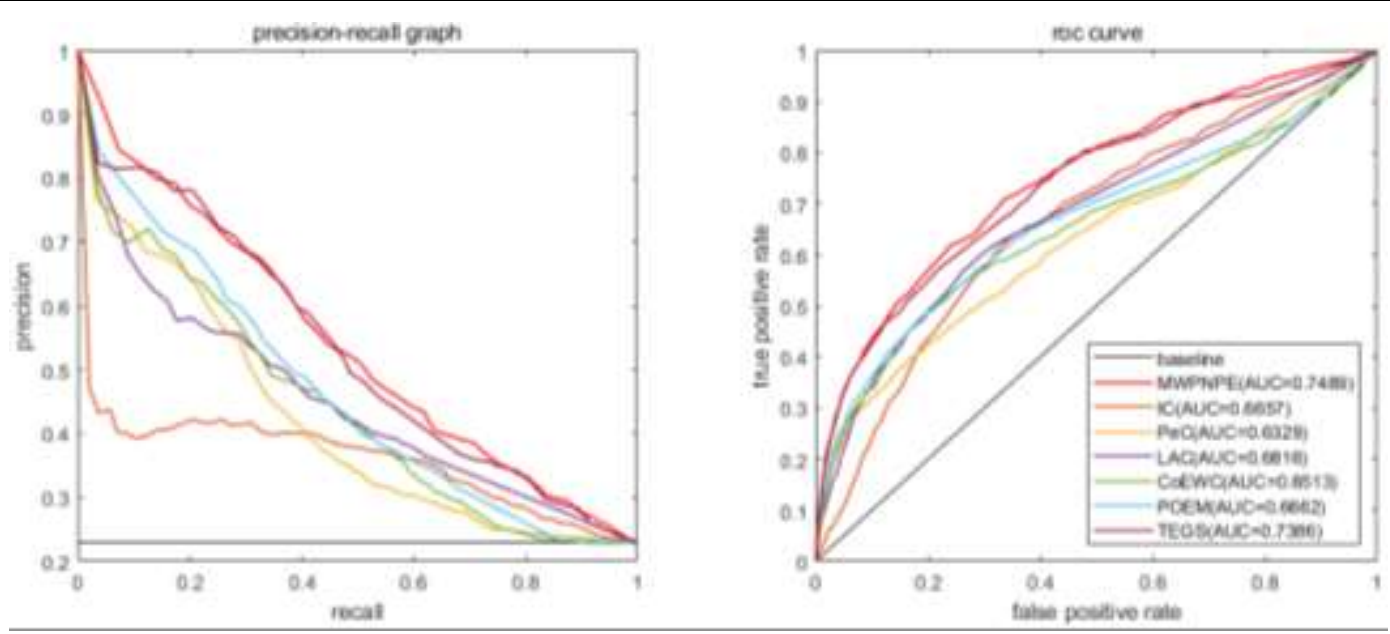

(b)

Fig.6 PR curves and ROC curves of MWPNPE and 12 competitive methods under the DIP database. (a) Comparison of PR curves and ROC curves between MWPNPE and SC, EC, DC, BC, CC, NC. (b) Comparison of PR curves and ROC curves between MWPNPE and IC, PEC, LAC, CoEWC, POEM, TEGS.

347 Table 3. Comparison of AUC values between MWPNPE and 12 competitive methods based on the 348 DIP database

\begin{tabular}{llllllll}
\hline method & MWPNPE & SC & EC & DC & BC & CC & NC \\
AUC & 0.7489 & 0.6384 & 0.6384 & 0.6704 & 0.6250 & 0.6291 & 0.6879 \\
\hline method & MWPNPE & IC & PeC & LAC & CoEWC & POEM & TEGS \\
& & & & & & & \\
AUC & 0.7489 & 0.6657 & 0.6329 & 0.6816 & 0.6513 & 0.6662 & 0.7386 \\
\hline
\end{tabular}


a
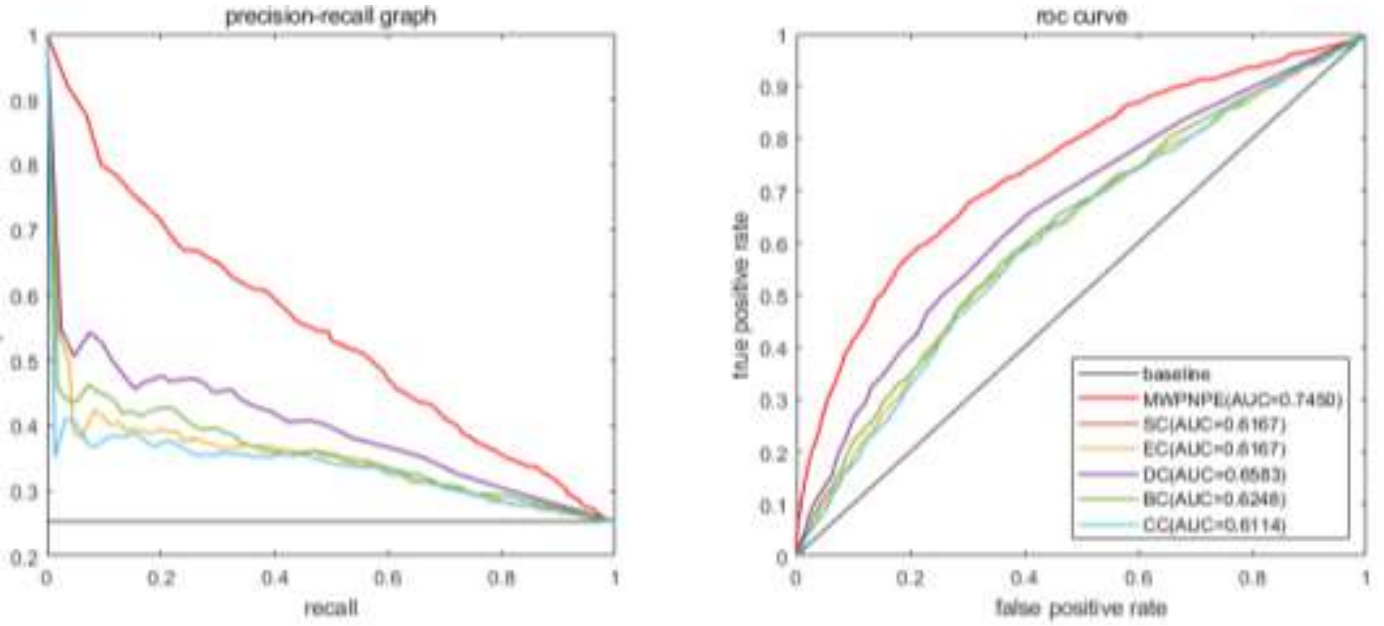

(a)
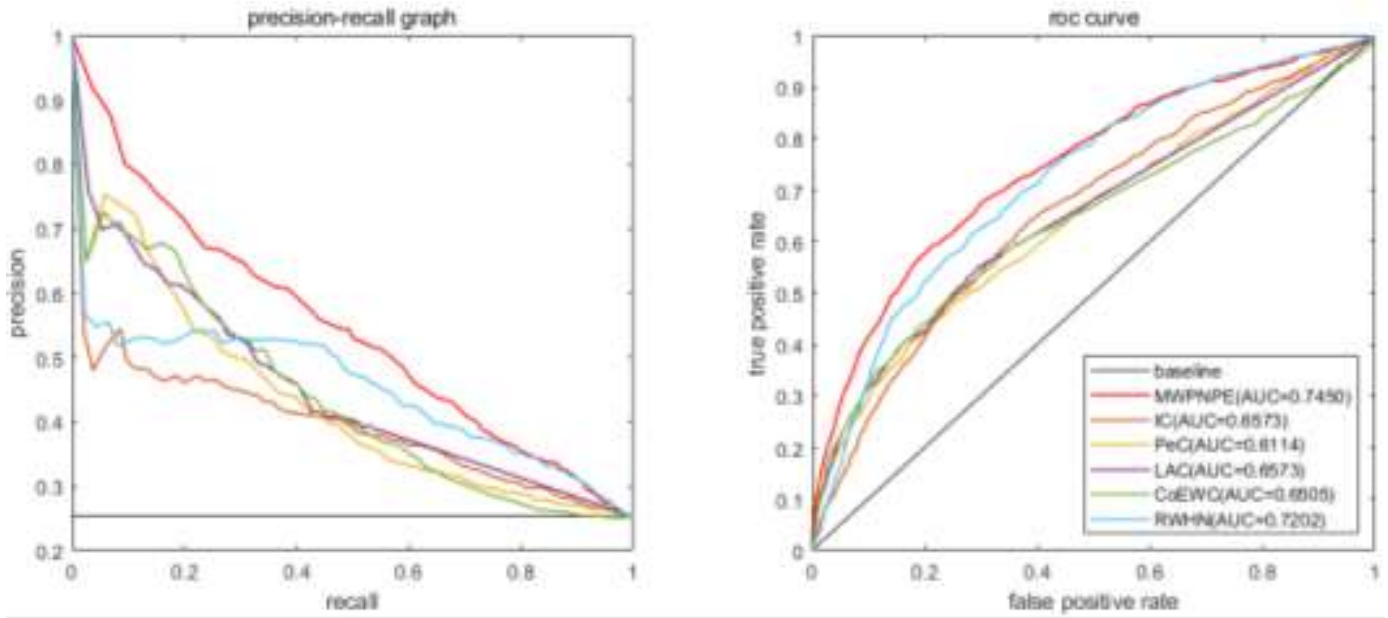

(b)

Fig.7 PR curves and ROC curves of MWPNPE and 10 competitive methods under the Krogan database. (a) Comparison of PR curves and ROC curves between MWPNPE and SC, EC, DC, BC, CC. (b) Comparison of PR curves and ROC curves between MWPNPE and IC, PEC, LAC, CoEWC, RWHN. 
351 Table 4. Comparison of AUC values between MWPNPE and 10 competitive methods based on the

352 Krogan database

\begin{tabular}{lllllll}
\hline method & MWPNPE & SC & EC & DC & BC & CC \\
AUC & 0.7450 & 0.6167 & 0.6167 & 0.6583 & 0.6248 & 0.6114
\end{tabular}

\begin{tabular}{llllll}
\hline method & MWPNPE & IC & PeC & CoEWC & RWHN
\end{tabular}
AUC
0.7450
0.6573
0.6446
0.6505
0.6396
0.7202

Effect of parameters on Performance of MWPNPE

In MWPNPE, we set a parameter $\tau$ with value interval of $(0,1)$ to adjust the final predicted criticality scores of proteins. During experiments, all possible values of $\tau$ will be used to try to obtain

356 the most effective and stable protein score. Among them, the setting value with the highest

357 prediction accuracy of essential protein will be selected as the final value of $\tau$. For simplicity, we

358 will set $\tau$ to $0.1, \ldots, 0.8$ and 0.9 under the Gavin database, the Krogan database and the DIP database

359 respectively to predict essential proteins, and illustrate the results in the following Table 5, Table 6

360 and Table 7 separately. From observing Table 5, it is easy to see that, under the Gavin database,

361 MWPNPE can achieve the maximum performance when $\tau$ is set to 0.8 or 0.9 . From observing Table

3626 , based on the Krogan database, MWPNPE can achieve the maximum performance when $\tau$ is equal

363 to 0.9 . From observing Table 7 , it is easy to see that, based on the DIP database, MWPNPE can

364 achieve the maximum performance when $\tau$ is equal to 0.8 or 0.9 . Thus, after comprehensive 
365 consideration, we will respectively set $\tau$ to $0.8,0.9$ and 0.8 in the experiments based on the databases

366 of Gavin, Krogan and DIP to compare MWPNPE with those advanced competitive models.

367 Table 5. Effect of the parameters $\tau$ on MWPNPE under the Gavin database

\begin{tabular}{|c|c|c|c|c|c|c|c|c|c|}
\hline & 0.1 & 0.2 & 0.3 & 0.4 & 0.5 & 0.6 & 0.7 & 0.8 & 0.9 \\
\hline Top50 & 42 & 44 & 44 & 44 & 44 & 45 & 45 & 45 & 44 \\
\hline Top 100 & 80 & 83 & 87 & 89 & 88 & 86 & 86 & 84 & 85 \\
\hline Top200 & 149 & 152 & 157 & 160 & 164 & 166 & 165 & 166 & 165 \\
\hline Top300 & 209 & 215 & 218 & 228 & 230 & 233 & 232 & 232 & 232 \\
\hline Top400 & 263 & 271 & 277 & 284 & 286 & 281 & 290 & 290 & 291 \\
\hline Top500 & 320 & 326 & 324 & 329 & 332 & 335 & 338 & 339 & 341 \\
\hline
\end{tabular}

369 Table 6. Effect of the parameters $\tau$ on MWPNPE under the Krogan database

\begin{tabular}{|c|c|c|c|c|c|c|c|c|c|}
\hline$\alpha$ & 0.1 & 0.2 & 0.3 & 0.4 & 0.5 & 0.6 & 0.7 & 0.8 & 0.9 \\
\hline Top50 & 40 & 42 & 42 & 43 & 43 & 44 & 44 & 45 & 45 \\
\hline
\end{tabular}




\begin{tabular}{lccccccccc}
\hline Top100 & 73 & 74 & 75 & 76 & 78 & 80 & 80 & 80 & 82 \\
Top200 & 142 & 145 & 147 & 147 & 146 & 146 & 149 & 148 & 148 \\
Top300 & 202 & 203 & 204 & 208 & 212 & 210 & 211 & 209 & 208 \\
Top400 & 257 & 261 & 262 & 264 & 263 & 265 & 266 & 262 & 266 \\
& & & & & & & & & \\
Top500 & 305 & 308 & 310 & 313 & 315 & 316 & 316 & 317 & 314 \\
\hline
\end{tabular}

370

371 Table 7. Effect of the parameters $\tau$ on MWPNPE under the DIP database

\begin{tabular}{|c|c|c|c|c|c|c|c|c|c|}
\hline & 0.1 & 0.2 & 0.3 & 0.4 & 0.5 & 0.6 & 0.7 & 0.8 & 0.9 \\
\hline Top100 & 74 & 78 & 77 & 80 & 82 & 83 & 83 & 86 & 85 \\
\hline Top200 & 139 & 148 & 151 & 155 & 156 & 160 & 160 & 160 & 161 \\
\hline Top30 & 201 & 207 & 211 & 217 & 222 & 224 & 224 & 224 & 227 \\
\hline Top400 & 257 & 262 & 267 & 274 & 279 & 284 & 288 & 288 & 288 \\
\hline Top500 & 311 & 310 & 317 & 320 & 327 & 333 & 337 & 340 & 344 \\
\hline Тор600 & 361 & 364 & 366 & 373 & 379 & 385 & 388 & 392 & 392 \\
\hline
\end{tabular}




\section{Conclusions}

374 Essential proteins play an important role in the process of cell life. The loss of essential proteins

375 will lead to the loss of cell function, and even lead to the inability of cells to survive. In recent years,

376 many potential key protein prediction methods based only on PPI network structure have been

377 developed. With the increasing improvement of PPI data and biological data and the development of

378 high-throughput technology, more and more methods based on the combination of PPI network

379 topology and biological information have been designed. However, they simply use the data of PPI

380 combined with biological information, which is not conducive to the realization of prediction

381 accuracy. Inspired by this, in this study, an iterative model named MWPNPE was proposed for

382 identifying essential proteins based on the whole process network of protein evolution. Comparing

383 with traditional state-of-the-art predictive models, MWPNPE can achieve better performance in

384 prediction of potential essential proteins. In the future, we will introduce more biological information

385 of proteins such as the subcellular location information and domain interaction information of protein

386 into MWPNPE to further improve its recognition ability.

\section{Abbreviations}

388 SC: Subgraph Centrality; EC: Eigenvector Centrality; DC: Degree Centrality; BC: Betweenness

389 Centrality; CC: Closeness Centrality; NC: Neighbor Centrality; IC: Information Centrality; LAC:

390 Local Average Connection; CoEWC: Co-Expression Weighted by Clustering coefficient; RWHN:

391 Randomly Walking in the Heterogeneous Network; PPI: Protein-Protein Interaction

\section{Acknowledgements}

394 Not applicable 


\section{Author Contributions}

396 YCZ and LW conceived the study. XL, ZZ and ZPC improved the study based on the original model.

397 ZPC, ZZ and LW supervised the study. YCZ and LW wrote the manuscript of the study. All authors 398 reviewed and improved the manuscript.

400 Funding Information

401 This work was supported by the National Natural Science Foundation of China under Grants 40261873221.

403

404 Availability of data and materials

405 All data generated or analysed during this study are included in this published article and its supplem 406 entary information files.

408 Declarations

409 Ethics approval and consent to participate

410 Not applicable.

411 Consent for publication

412 Not applicable.

413 Competing interests

414 The authors declare that they have no competing interests.

416 Author details

$417{ }^{1}$ Institute of Bioinformatics Complex Network Big Data, Changsha University, Changsha 410022, 418 China. ${ }^{2}$ Big Data Innovation and Entrepreneurship Education Center of Hunan Province, Changsha 419 University, Changsha 410022, China 


\section{References}

421 [1] Divya M, Wise R P, Dickerson J A, et al. DiffSLC: A graph centrality method to detect essential 422 proteins of a protein-protein interaction network[J]. Plos One, 2017, 12(11): e0187091.

423 [2] Giaever G, Chu A M, Ni L, et al. Functional profiling of the Saccharomyces cerevisiae 424 genome[J]. Nature, 2002, 418(6896): p.387-391.

425 [3] Kamath RS, Fraser AG, Dong Y, Poulin G, Durbin R, Gotta M, Kanapin A, Le Bot N, Moreno S, 426 Sohrmann M, et al. Systematic functional analysis of the Caenorhabditis elegans genome using rnai.

427 Nature. 2003;421(6920):231-7.

428 [4] Cullen L M, Arndt G M. Genome-wide screening for gene function using RNAi in mammalian 429 cells[J]. Immunology \& Cell Biology, 2005, 83(3): 217.

430 [5] Dai W, Chen B, Peng W, et al. A Novel Multi-Ensemble Method for Identifying Essential 431 Proteins[J]. Journal of Computational Biology: a Journal of Computational Molecular Cell Biology, $4322021,28(7)$.

433 [6] Zhang W, Xue X, Xie C, et al. CEGSO: Boosting Essential Proteins Prediction by Integrating 434 Protein Complex, Gene Expression, Gene Ontology, Subcellular Localization and Orthology 435 Information[J]. Interdisciplinary Sciences: Computational Life Sciences, 2021:1-13.

436 [7] Estrada E, Rodriguez-Velazquez J A. Subgraph Centrality in Complex Networks[J]. Physical 437 Review E Statistical Nonlinear \& Soft Matter Physics, 2005, 71(5 Pt 2):056103.

438 [8] Bonacich, Phillip. Power and Centrality: A Family of Measures[J]. American Journal of 439 Sociology, 1987, 92(5):1170-1182. 
440 [9] Hahn M W, Kern A D. Comparative Genomics of Centrality and Essentiality in Three Eukaryotic 441 Protein-Interaction Networks[J]. Molecular Biology \& Evolution, 2005(4):803-6.

442 [10] Poulo J M, Amy B, Ingber D E, et al. High-Betweenness Proteins in the Yeast Protein 443 Interaction Network[J]. Journal of Biomedicine \& Biotechnology, 2005.

444 [11] Wuchty S, Stadler P F. Centers of complex networks[J]. Journal of Theoretical Biology, 2003, $445 \quad 223(1): 45-53$.

446 [12] Wang J, Li M, Wang H, et al. Identification of Essential Proteins Based on Edge Clustering 447 Coefficient[J]. IEEE/ACM Transactions on Computational Biology \& Bioinformatics, 2012, 448 9(4):1070-1080.

449 [13] Zelen S M. Rethinking centrality: Methods and examples[J]. Social Networks, 1989.

450 [14] Qi Yi, Luo Jiawei. Prediction of Essential Proteins Based on Local Interaction Density [J]. 451 IEEE/ACM Trans on Computational Biology and Bioinformatics, 2016. 13 (6): 1170-1182.

452 [15] Li M, Lu Y, Wang J, et al. A Topology Potential-Based Method for Identifying Essential 453 Proteins from PPI Networks[J]. IEEE/ACM Transactions on Computational Biology \& 454 Bioinformatics, 2015, 12(2):372.

455 [16] Chung-Yen L, Chia-Hao C, Hsin-Hung W, et al. Hubba: hub objects analyzer-a framework of 456 interactome hubs identification for network biology[J]. Nucleic Acids Research, 2008, 36(suppl_2): 457 W438.

458 [17] Jeong H M, Mason S P, AL Barabási, et al. Lethality and centrality in protein networks[J]. 459 Nature, 2001, 411(6833):41-2. 
460 [18] Sprinzak E, Sattath S, Margalit H. How reliable are experimental protein-protein interaction 461 data? J Mol Biol. 2003;327(5):919-23.

462 [19] Kuchaiev O, Ra?Ajski M , Higham D J , et al. Geometric De-noising of Protein-Protein 463 Interaction Networks[J]. Plos Computational Biology, 2009, 5(8): e1000454.

464 [20] Fengyu Zhang, Wei Peng, Yunfei Yang, Wei Dai,Junrong Song. A Novel Method for 465 Identifying Essential Genes by Fusing Dynamic Protein-Protein Interactive Networks. Genes. 2019, $46610(1), 31$.

467 [21] Lei X, Yang X, Wu F-X. Artificial fish swarm optimization-based method to identify essential 468 proteins. IEEE/ACM Trans Comput Biol Bioinform. 2018;17(2):495-505.

469 [22] Zhao B, Wang J, Li M, Wu FX, Pan Y. Prediction of essential proteins based on overlapping 470 essential modules. IEEE Trans NanoBiosci. 2014;13(4):415-24.

471 [23] Xue Z, Xu J, Xiao WX. A new method for the discovery of essential proteins. PLoS ONE. $472 \quad 2013 ; 8:$ e58763.

473 [24] Ren R, Wang J, Li M, et al. Prediction of essential proteins by integration of PPI network 474 topology and protein complexes. Bioinformatics research and applications. Berlin, Heidelberg: 475 Springer 2011; pp. 12-24. http://dx.doi.org/10.1007/978-3-642-21260-4-6.

476 [25] Min L, Zhang H, Wang JX, Yi P. A new essential protein discovery method based on the 477 integration of protein-protein interaction and gene expression data. BMC Syst Biol. 2012; 6:1-9

478 [26] Sun W, Wang L, Peng J, et al. A Cross-Entropy-based Method for Essential Protein 479 Identification in Yeast Protein-Protein Interaction Network[J]. Current Bioinformatics, $2020,15$. 
[27] Li S, Chen Z, X He, et al. An Iteration Method for Identifying Yeast Essential Proteins from

481 Weighted PPI Network based on topological and functional features of proteins[J]. IEEE Access, $482 \quad 2020$, PP (99):1-1.

483 [28] Peng W, Wang J, Wang W, Liu Q, Wu F-X, Pan Y. Iteration method for predicting essential 484 proteins based on orthology and protein-protein interaction networks. BMC Syst Biol. 2012;6(1):148517.

[29] Zhang W, Xu J, Li Y, Zou X. Detecting essential proteins based on network topology, gene 487 expression data, and gene ontology information. IEEE/ACM Trans Comput Biol Bioinform. 488 2016;15(1):109-16.

[30] Zhang $\mathrm{W}, \mathrm{Xu} \mathrm{J}$, Zou X. Predicting essential proteins by integrating network topology, 490 subcellular localization information, gene expression profile and go annotation data. IEEE/ACM 491 Trans Comput Biol Bioinform. 2019;17(6):2053-61.

492 [31] Lei X, Zhao J, Fujita H, Zhang A. Predicting essential proteins based on RNA-seq, subcellular 493 localization and go annotation datasets. Knowl Based Syst. 2018; 151:136-48.

494 [32] B. Zhao, Y. Zhao, X. Zhang, Z. Zhang, F. Zhang, and L. Wang, "An iteration method for 495 identifying yeast essential proteins from heterogeneous network," BMC Bioinf., vol. 20, no. 1, p. $496 \quad 355$, Dec. 2019.

497 [33] Dai C, He J, Hu K, et al. Identifying essential proteins in dynamic protein networks based on an 498 improved h -index algorithm[J]. BMC Medical Informatics and Decision Making, 2020, 20(1):1-10.

499 [34] Gavin A-C, Aloy P, Grandi P, Krause R, Boesche M, Marzioch M, Rau C, Jensen LJ, Bastuck S, 500 Dümpelfeld B, et al. Proteome survey reveals modularity of the yeast cell machinery. Nature. $501 \quad 2006 ; 440(7084): 631-6$. 
503 Global landscape of protein complexes in the yeast saccharomyces cerevisiae. Nature. $5042006 ; 440(7084): 637-43$.

505 [36] Xenarios I, Salwinski L, Duan XJ, Higney P, Kim S-M, Eisenberg D. Dip, the database of 506 interacting proteins: a research tool for studying cellular networks of protein interactions. Nucleic 507 Acids Res. 2002;30(1):303-5.

508 [37] Saccharomyces Genome Deletion Project. http:// yeast delet ion. stanf ord. edu/.

509 [38] Cherry JM, Adler C, Ball C, Chervitz SA, Dwight SS, Hester ET, Jia Y, Juvik G, Roe T, 510 Schroeder M, et al. Sgd: saccharomyces genome database. Nucleic Acids Res. 1998;26(1):73-9.

511 [39] Zhang R, Lin Y. Deg 5.0, a database of essential genes in both prokaryotes and eukaryotes. 512 Nucleic Acids Res. 2009;37(suppl_1):455-8.

513 [40] Mewes H-W, Frishman D, Mayer KF, Münsterkötter M, Noubibou O, Pagel P, Rattei T,

514 Oesterheld M, Ruepp A, Stümpflen V. Mips: analysis and annotation of proteins from whole 515 genomes in 2005. Nucleic Acids Res. 2006;34(suppl_1):169-72.

516 [41] Östlund G, Schmitt T, Forslund K, Köstler T, Messina DN, Roopra S, Frings O, Sonnhammer

517 EL. Inparanoid 7: new algorithms and tools for eukaryotic orthology analysis. Nucleic Acids Res. 518 2010;38(suppl_1):196-203.

519 [42] Tu BP, Kudlicki A, Rowicka M, McKnight SL. Logic of the yeast metabolic cycle: temporal 520 compartmentalization of cellular processes. Science. 2005;310(5751):1152-8. 
521 [43] Holman AG, Davis PJ, Foster JM, Carlow CK, Kumar S. Computational prediction of essential 522 genes in an unculturable endosymbiotic bacterium, Wolbachia of Brugia malayi. BMC Microbiol. $5232009 ; 9(1): 243$.

524 [44] Davis J. The Relationship Between Precision-Recall and ROC Curves[C]// Proceedings of the 525 23th International Conference on Machine Learning, 2006. 2006.

526 [45] D. Horyu, T. Hayashi. Comparison between pearson correlation coefficient and mutual 527 information as a similarity measure of gene expression profiles, Jpn. J. Biometrics, vol. 33, no. 2, pp. 528 125-143, 2013. 